\title{
Utility of chemoradioselection for the optimization of treatment intensity in advanced hypopharyngeal and laryngeal carcinoma
}

\author{
MUNEYUKI MASUDA ${ }^{1}$, TAKAHIRO WAKASAKI ${ }^{1}$, SATOSHI TOH $^{1}$, YOSHINORI UCHIDA ${ }^{1}$, \\ HIDEFUMI RIKIMARU $^{1}$, NAONOBU KUNITAKE ${ }^{2}$ and YUICHIRO HIGAKI ${ }^{1}$ \\ Departments of ${ }^{1} \mathrm{Head}$ and Neck Surgery, and ${ }^{2}$ Radiology, National Kyushu \\ Cancer Center, Minamiku, Fukuoka 811-1395, Japan
}

Received June 26, 2017; Accepted October 19, 2017

DOI: $10.3892 /$ mco.2017.1466

\begin{abstract}
Definitive concomitant chemoradiotherapy (CRT) with high-dose cis-platinum (CDDP) is a current standard protocol for advanced laryngeal and hypopharyngeal cancer sparing surgery for salvage. However, this modality is associated with limited feasibility and frequent sever toxicities. In the present study, a 'chemoradioselection' protocol with minimal toxicity was developed using initial response to CRT as a biomarker for patient selection. Between 2000, March and 2012, September 123 patients with stage III (44), IV (79) laryngeal (64) and hypopharyngeal carcinoma (59) excluding T4 cases were enrolled to this protocol. Two cycles of split $\left(15 \mathrm{mg} / \mathrm{m}^{2} \times 5\right.$ days, 2000-2008) or bolus (80 mg/m², 2009-present) CDDP was concurrently administered. Tumor responses were evaluated after $40 \mathrm{~Gy}$ of CRT and 64 responders (chemoradioselected, CRS) received further CRT up to $70 \mathrm{~Gy}$, while radical surgery was recommended for the 59 non-responders (N-CRS), and 34 underwent surgery (N-CRS-ope). The remaining 25 patients who refused surgery (N-CRS-refu) were treated with continuous CRT. The 5-year overall survival (OS) and disease-specific survival (DSS) were 67, and 77\%, respectively. The CRS demonstrated favorable 5-year OS (73\%) and laryngo-esophageal dysfunction-free survival (LEDFS, 69\%) rates. In contrast, the N-CRS-refu showed significantly lower 5-year OS (47\%) compared with CRS (73\%) and N-CRS-ope (70\%) (P=0.0193), and significantly lower 5-year LEDFS (20\%) compared with the CRS $(69 \%)(\mathrm{P}<0.0001)$. On multivariate analyses, including $\mathrm{T}, \mathrm{N}$, primary site and planned treatment (CRS + N-CRS-ope) or not (N-CRS-refu), unplanned treatment alone showed a significant correlation with poor OS [hazard ratio (HR),
\end{abstract}

Correspondence to: Dr Muneyuki Masuda, Department of Head and Neck Surgery, National Kyushu Cancer Center, 3-1-1, Notame, Minamiku, Fukuoka 811-1395, Japan

E-mail: masuda.m@nk-cc.go.jp

Key words: chemoradioselection, laryngo-esophageal dysfunction free survival, optimization of therapeutic intensity, toxicities, survival benefit of surgery
2.584; 95\% confidence interval (CI), 1.313-4.354; $\mathrm{P}=0.007)$. Chemoradioselection reflects the biological aggressiveness of each tumor, and is able to segregate patients for functional laryngeal preservation with moderate intensity CRT (150-160 $\mathrm{mg} / \mathrm{m}^{2}$ of CDDP) from those who would be better treated with surgery. This strategy may be useful for the optimization of the therapeutic intensity.

\section{Introduction}

The trend in the treatments for advanced head and neck squamous cell carcinoma (HNSCC) has shifted from radical surgery to organ preservation due, in large part, to the two milestone studies conducted in the 1990s: The Department of Veterans Affairs Laryngeal Cancer Study Group (VALCSG) (1) and European Organization for Research and Treatment of Cancer (EORTC) 24891 (2). These two studies clearly demonstrated that laryngeal preservation is feasible with combined use of induction chemotherapy and radiation without compromising patients' survival in advanced laryngeal and hypopharyngeal carcinoma. As a result, organ-preservation was adopted as the main goal of the clinical studies and whereby unprecedented dose-intensification has been conducted mainly through two forms of modalities: Concurrent chemoradiotherapy (CRT) (e.g., clinical trials led by the Radiation Therapy Oncology Group [RTOG]) or sequential therapy (ST) composed of induction chemotherapy and CRT (e.g., GORTEC and Tax 324 protocols) (3-6). Because of the further improved laryngeal preservation, these dose-intensified organ-preserving strategies (DIOPSs) are currently proposed as the standard for organ preservation $(7,8)$. However, it is becoming apparent that DIOPSs involve critical issues. Firstly, only a limited number of advanced HNSCC patients benefit from DIOPSs, because these types of experimental therapies are feasible only in a select subset of patients (i.e., patients with good general condition) who can tolerate these heavy regimens. This limitation may be related to the recent surprising results of large surveys based on the Surveillance, Epidemiology, and End Results (SEER) or the National Cancer Data Base (NCDB), which demonstrated a worsening survival trend in patients with laryngeal cancer and only a marginal improvement in those with hypopharyngeal cancer $(9,10)$. Secondly, it is obvious that current DIOPSs have reached the upper limit 
of human tolerance in terms of late toxicitiy, as exemplified in the recently published long-term results of RTOG 91-11. On this regimen, which employs concurrent $100 \mathrm{mg} / \mathrm{m}^{2}$ of CDDP tri-weekly, as much as $43 \%$ of the patients with preserved larynx developed laryngo-esophageal dysfunction, and which eventually accounted for the high rate of tumor-unrelated deaths (11-13). Thirdly, it became practically infeasible to compare the treatment results of radical surgery with organ-preserving treatments in randomized control studies, due to the strong dogma: Similar survival is achievable by either DIOPS or radical surgery on the basis of VALCSG and EORTC 24891 studies $(1,2)$ conducted more than 20 years ago. Consequently, the survival benefit of radical surgery appears to have been overly underestimated despite considerable technical advancements and the improved multimodality setting in which surgery is conducted.

Contrary to the trend of DIOPSs, we have treated HNSCC patients using a distinctive platform, in which 30-40 GY of induction CRT is used as a selection tool for organ preservation $(14,15)$. In this algorithm-based 'chemoradioselection' strategy, only patients who demonstrate good response to induction CRT (i.e., 'chemoradioselected': CRS), proceed to organ preservation arm and then receive further CRT up to 60-70 Gy. For the remaining non-responders (i.e., 'non-chemoradioselected': N-CRS), radical surgery is recommended. Mainly using moderate intensity CRT regime, we obtained quite satisfactory laryngeal preservation and survival in T2 glottic carcinoma and overall survival in oropharyngeal carcinoma with minimal toxicity (14,16-18). In a recent pilot study on advanced hypopharyngeal carcinoma (19), we reported the utility of this protocol; chemoradioselection can segregate tumors for organ-preservation with use of moderate intensity CRT from those that would be better treated by radical surgery. Interestingly irrespective of clinical stage, CRS patients demonstrated quite favorable survival and organ preservation with moderate intensity CRT that seldom caused laryngo-esophageal-dysfunction, whereas radical surgery appeared to exhibit survival benefit in the N-CRS. In this context, the aim of this study is to verify the utility of this strategy in a larger scale study employing patients with advanced hypopharyngeal and laryngeal cancer and to explore the potential of this strategy as a novel platform for the treatment of advanced HNSCC which may address the issues associated with the current DIOPSs mentioned above.

\section{Patients and methods}

Patients eligibility and protocol. All patients had pathologically confirmed, previously untreated stage III or IV laryngeal and hypopharyngeal cancer according to the UICC TNM classification(2002 and 2007). Clinical staging and the diagnosis of double cancer were done by endoscopy, contrast enhanced computed tomography, magnetic resonance imaging, ultrasonography, and fluorodeoxyglucose positron emission tomography. Patients who had distant metastasis and/or synchronous cancer were excluded. T4 cases were also excluded, because use of organ preserving treatment in this stage is controversial, especially for hypopharyngeal tumors (20,21). All patients were required to have an Eastern Cooperative Oncology Group Organization performance status $\leqq 2$, and adequate medical and laboratory parameters required for CRT. The choice of platinum agent was determined by the creatinine clearance (Ccr): Cis-platin (CDDP) was used for those with $\mathrm{Ccr} \geqq 60 \mathrm{ml} / \mathrm{min}$ and palapalatin (CBDCA) for those with $\mathrm{Ccr}<60 \mathrm{ml} / \mathrm{min}$. Enrolled patients were treated according to the algorithm-based chemoradioselection protocol. From 2000 to 2008, split CDDP (15 mg/m²/day) or CBDCA ( $\mathrm{AUC}=1 /$ day) was administered from day 1 to 5 , concomitantly with external beam of irradiation (2.0 Gy/day); since $2009 \mathrm{CDDP}\left(80 \mathrm{mg} / \mathrm{m}^{2} /\right)$ or CBDCA (AUC=5) on day 1 was administered. After $40 \mathrm{~Gy}$ of CRT, the tumor response was evaluated either clinically or pathologically. With the split CDDP regimen, complete response at the primary site was considered as chemoradioselected (CRS). For patients treated with bolus CDDP, $\geq 50 \%$ reduction of primary tumor was used as the criteria for CRS (Fig. 1). The CRS patients continued to receive additional CRT up to $70 \mathrm{GY}$ and received planned neck dissection (PND) for residual N. For the N-CRS patients, radical surgery was recommended and conducted (N-CRS-ope), when consent for total laryngectomy was obtained. However, to those who refused surgery (N-CRS-refu), continuous CRT was administered up to $70 \mathrm{GY}$. This algorithm-based protocol was approved by the Institutional Review Board and all patients gave documented informed consent.

Patients characteristics. From 2000, March to 2012, September 123 patients with stage III and IV HPC were registered for this algorithm-based chemoradioselection protocol. All 123 patients were followed up for more than 36 months; median follow up period was 63 months (range 4-168). Their average age was 64 . The patient characteristics are summarized in Table 1.

Adverse effects. Adverse effects were evaluated according to the Common Terminology Criteria for Adverse Events version 3.0.

End-points and statistical analyses. The primary endpoints were overall survival (OS) and laryngo-esophageal dysfunction free survival (LEDFS). Use of LEDFS as an endpoint was proposed by the Larynx Preservation Consensus Panel in 2009, in which, death, local recurrence, total or partial laryngectomy, tracheostomy, and feeding tube/gastrostomy insertion after $\geqq 2$ years were considered as a composite event (22). Thus, LEDFS reflects the 'functional' laryngeal preservation in surviving patients more accurately than laryngectomy free survival (LFS) that has been used conventionally. In order to clarify the efficacy of chemoradioselection, OS and LEDFS in specific subgroups of patients (e.g., treatment courses and clinicopathological factors) were also calculated. For these analyses, Kaplan-Meier curves were generated and the between-group differences were assessed by log-rank test for statistical significance. The cause of death and the results of laryngeal preservation were assessed by Chi-square test or Fisher's exact test. Multivariate Cox proportional hazard model were used to calculate the effects of $\mathrm{T}, \mathrm{N}$, primary sites and treatment courses on OS. Values of $\mathrm{P}<0.05$ were considered statistically significant.

\section{Results}

Treatment courses. The treatment courses of patients are demonstrated in Fig. 1. After 40 Gy of CRT, 64 (52\%) of patients 
Table I. Patient characteristics $(N=123)$.

\begin{tabular}{lc}
\hline Characteristic & No. of patients $(\%)$ \\
\hline Sex & $113(92)$ \\
Male & \\
Female & $10(8)$ \\
Primary site & \\
Hypopharynx & $59(48)$ \\
Larynx & $64(52)$ \\
T classification & \\
T1 & $13(11)$ \\
T2 & $58(47)$ \\
T3 & $52(42)$ \\
N classification & \\
N0 & $19(15)$ \\
N1 & $25(20)$ \\
N2a & $2(2)$ \\
N2b & $49(40)$ \\
N2c & $19(15)$ \\
N3 & $9(7)$ \\
TNM Stage & $44(36)$ \\
III & \\
IVa & $70(57)$ \\
IVb & $9(7)$ \\
\hline
\end{tabular}

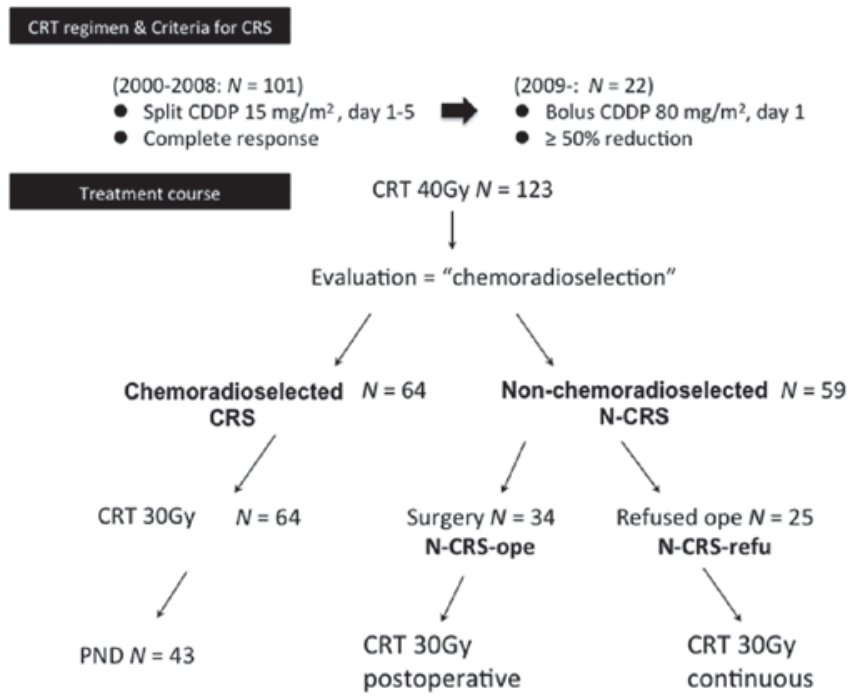

Figure 1. Algorithm and treatment course of patients. In brief, tumor responses to the initial $40 \mathrm{~Gy}$ of CRT are evaluated according to the distinctive criteria shown in the Figure. Good responders (chemoradioselected: CRS) procced to undergo further $30 \mathrm{~Gy}$ of CRT and planned neck dissection (PND) for residual cervical lymph nodes. For poor responders (non-chemoradieselected: N-CRS), radical surgery is conducted when consent is obtained (N-CRS-ope), while continuous CRT is administered for patients who refused surgery (N-CRS-refu).

were classified as CRS, while 59 (48\%) of patients as N-CRS. CRS patients were further treated by additional $30 \mathrm{~Gy}$ of CRT and 43 patients received PND. Among the N-CRS population, 34 patients underwent radical surgery (N-CRS-ope), while
25 patients refused operation (N-CRS-refu). Additional 30 Gy of CRT was given either as postoperatively to N-CRS-ope or in continuation to N-CRS-refu. There were 101 patients who received split CDDP, while 22 patients received bolus CDDP.

Adverse effects. None of the patients developed grade 5 toxicity. Only one patient developed grade 4 toxicity (granulocytopenia and neutropenia). Cumulative grade 3 adverse effects included leucopenia $(N=13)$; neutropenia $(N=6)$; thrombocytopenia $(N=3)$; dermatitis $(N=4)$; mucositis $(N=13)$; anolexia $(N=1)$; pneumonia $(N=2)$; dysphagia $(N=5)$; renal dysfunction $(N=1)$; and liver dysfunction $(N=1)$. Comparable rates of adverse effects ( $\geq$ grade 3 ) were observed in the split CDDP (38\%) and bolus CDDP (43\%) groups.

Patients survival. There were a total of 50 deaths (21 in the CRS patients, 15 in the N-CRS-ope, and 14 in the N-CRS-refu groups). Out of the 21 deaths in the CRS arm, 7 were tumor-related deaths [loco-regional failure $(N=3)$; distant metastases $(N=4)$ ], while 14 were tumor-unrelated deaths (metachronous cancer $(N=4)$; pneumonia $(N=1)$; hepatitis $(N=1)$; and others $(N=8)$. Out of the 15 deaths in $\mathrm{N}$-CRS-ope arm, 7 were tumor-related deaths [loco-regional failure [N=3]; distant metastasis $(N=4)$ ] and 8 were tumor-unrelated deaths [metachronous cancer $(N=4)$; heart failure $(N=1)$; and pneumonia $(N=3)$ ]. Out of the 14 deaths in the N-CRS-refu arm, 11 were tumor-related deaths [loco-regional failure $(N=8)$; distant metastasis $(N=3)$ ] and 3 were tumor-unrelated deaths [metachronous cancer $(N=2)$; others $(N=1)]$. Thus, patients in the N-CRS-refu arm experienced significantly $(\mathrm{P}=0.0038$, Chi-square-test) higher tumor-related death rate $(11 / 25,44 \%)$ as compared to that in the CRS $(10 / 64,10 \%)$ and the N-CRS-ope $(7 / 34,20 \%)$ arms. The rate of distant metastasis in the N-CRS-ope $(4 / 34,11 \%)$ and the N-CRS-refu $(3 / 25,12 \%)$ arms was approximately 2 times higher than that in the CRS $(4 / 64,6 \%)$ arm, although the difference was not statistically significant. Collectively, the 5-year cumulative disease-specific survival rate (DSS) and OS rates were $77 \%$ and $65 \%$, respectively (Fig. 2). We then analyzed the effects of 4 candidate prognostic factors [T (T1, 2 vs. T3); $\mathrm{N}$ (N0, 1 vs. N2, 3); primary site (larynx vs. hypopharynx); and treatment [planned: i.e., CRS + N-CRS-ope vs. unplanned: i.e., N-CRS-refu] on OS using both univariate and multivariate models. The 5-year OS in the CRS (73\%) and the N-CRS-ope arms $(70 \%)$ was significantly better $(\mathrm{P}=0.0193)$ than that $(47 \%)$ in the N-CRS-refu arm. In other words, unplanned-treatment (i.e., NCR-refu) was associated with significantly $(\mathrm{P}=0.0061)$ worse 5-year OS (47\%) as compared to that associated with planned-treatment (i.e., CRS+N-CRS-ope arms, 72\%) (Fig. 3). In contrast, the Kaplan-Meier curves produced by T (T1, 2 vs. T3), $\mathrm{N}$ (N0, 1 vs. N2, 3), and primary site (larynx vs. hypopharynx) did not display significant differences. This tendency was also confirmed in the Cox proportional hazard model, in which only unplanned treatment (i.e., N-CRS-refu arm) showed a significant correlation with poor OS (HR: $2.583,95 \% \mathrm{CI}$ : $1.313-4.354$, $\mathrm{P}=0.007$ ) (Table II). We then additionally analyzed the hazard ratios of the 4 factors for OS according to the two different stratification criteria: Patients who received 70 Gy of CRT without initial laryngectomy (CRS + N-CRS-refu) and those who were N-CRS (N-CRS-ope + N-CRS-refu). In the former cohort, unplanned treatment (HR: 3.137, 95\% CI: 1.458-6.661, 

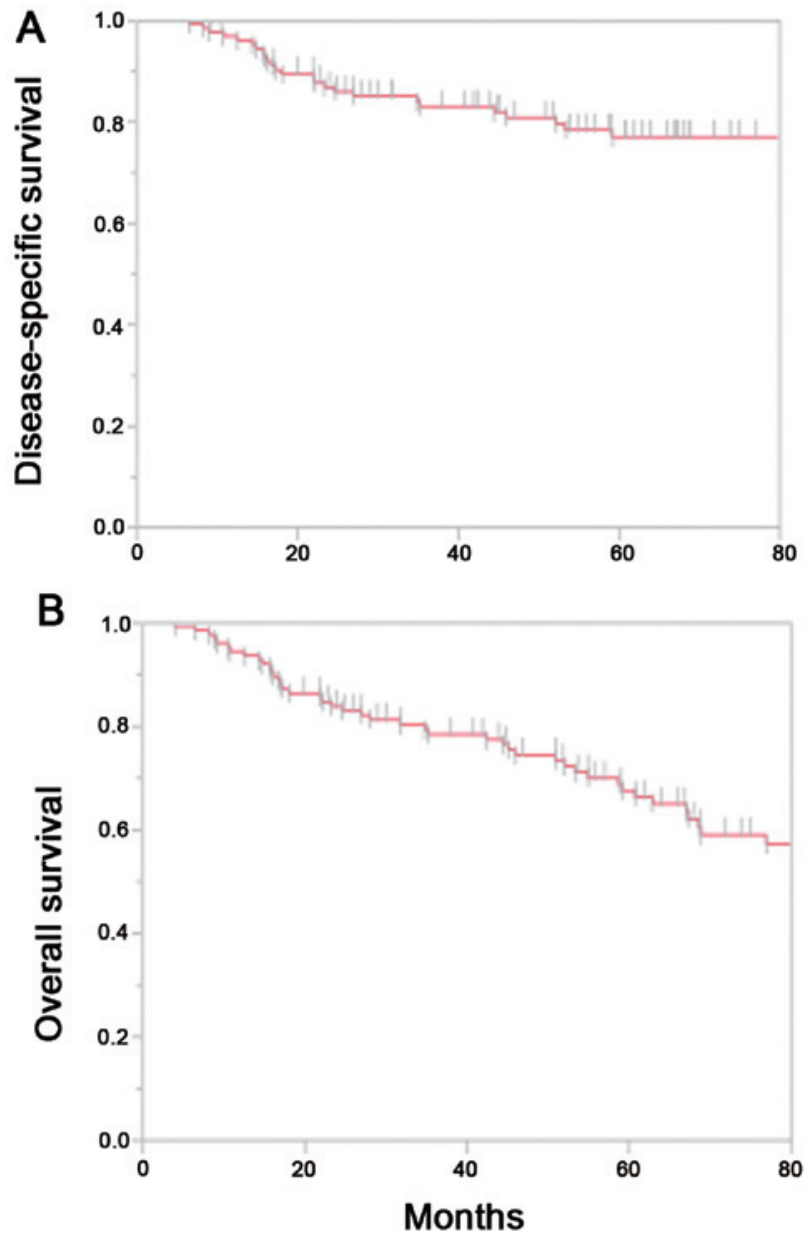

Figure 2. Disease-specific (A) and overall (B) survival curves.

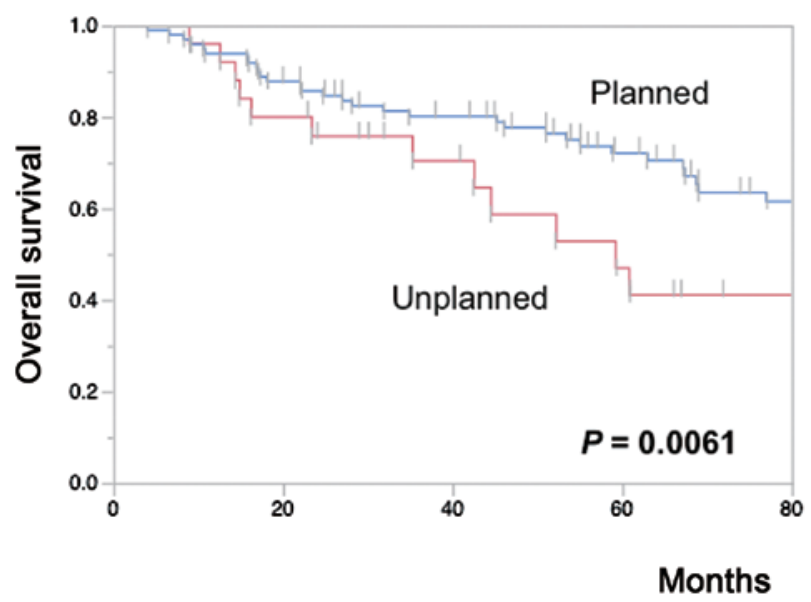

Figure 3. Overall survival curves according to the treatment courses.

$\mathrm{P}=0.0039)$ and hypopharyngeal tumor (HR: $2.458,95 \% \mathrm{CI}$ 1.137-5.392, $\mathrm{P}=0.0223$ ) were associated with significantly increased risk of death. In the latter cohort, only unplanned treatment was a significant risk factor (HR: $7.638,95 \% \mathrm{CI}$ : 2.294-27.542, $\mathrm{P}=0.0008)$.

Laryngeal preservation. Sixteen out of 64 (25\%) in the CRS arm developed tumor recurrences. Fourteen of these underwent
Table II. Hazard ratios (HR) using multivariate Cox proportional hazard model.

\begin{tabular}{|c|c|c|c|c|}
\hline \multirow[b]{2}{*}{ Class } & \multirow[b]{2}{*}{ Factor } & \multicolumn{3}{|c|}{ Multivariate analysis } \\
\hline & & HR & $95 \% \mathrm{CI}$ & P-value \\
\hline \multirow[t]{2}{*}{ Treatment } & Planned & Ref. & & \\
\hline & Unplanned & 2.583 & $1.313-4.854$ & 0.007 \\
\hline \multirow[t]{2}{*}{ T stage } & $1-2$ & Ref. & & \\
\hline & 3 & $\begin{array}{l}0.691 \\
0.2518\end{array}$ & $0.363-1.297$ & \\
\hline \multirow[t]{2}{*}{$\mathrm{N}$ stage } & $0-1$ & Ref. & & \\
\hline & $2-3$ & $\begin{array}{l}1.017 \\
0.9511\end{array}$ & $0.577-.1 .816$ & \\
\hline \multirow[t]{2}{*}{ Primary site } & Larynx & Ref. & & \\
\hline & Hypopharynx & $\begin{array}{l}0.584 \\
0.0949\end{array}$ & $0.306-1.097$ & \\
\hline
\end{tabular}

surgery including total laryngectomy, of which 9 were salvaged (i.e., survived), while 5 patients were died of disease unrelated to laryngeal or hypopharyngeal cancer. Thus, the surgical salvage rate in patients with recurrence was $64 \%(9 / 16)$. The $\mathrm{N}$-CRS-refu arm experienced significantly $(\mathrm{P}=0.0114$, Fisher's exact test) higher recurrence rate in the larynx and hypopharynx $(56 \%, 14 / 25)$ as compared to that in the CRS-group. Seven patients proceeded to surgery and 6 of these were salvaged, while one patient died of other cancer; the surgical salvage rate in this cohort was $43 \%$ (6/14). No laryngo-esophageal dysfunction was observed in the survivors with preserved larynx. Based on these data, the cumulative LEDF survival rate of all patients were $41 \%$ at 5 -years. The CRS arm demonstrated significantly $(\mathrm{P}<0.0001)$ better 5-year (69\%) LEDS than the N-CRS-refu arm (3.6\%). In the Kaplan-Meier curves produced by the group ( $\mathrm{T}$, $\mathrm{N}$, and primary site), the $\mathrm{T}$ classification alone had a significant effect on LEDFS; T3 tumors were associated with significantly $(\mathrm{P}=0.0012)$ lower LEDFS (25\%) than $\mathrm{T} 1$ and $\mathrm{T} 2$ tumors $(53 \%)$.

Split CDDP vs. bolus CDDP. Although preliminary, we examined whether bolus CDDP could potentate the efficacy of chemoradioselection over split CDDP. The bolus CDDP group demonstrated higher rates of CRS (68\%) as compared to that in the split CDDP group (51\%). The bolus group also showed a tendency for higher 3-year OS and LEDFS (90 and 64\%) than split CDDP (76\% and 45\%). In T1 and T2 cases, bolus CDDP improved the 5-year LEDFS from 61 to $80 \%$. However, surprisingly, the LEDFS of T3 in the bolus CDDP dropped sharply to $29 \%$ at 2-year post-treatment, which is rather worse than that of overall T3 data (38\%). These findings indicate that bolus CDDP may contribute to the improvement in overall survival and laryngeal preservation in T1and $\mathrm{T} 2$, but not $\mathrm{T} 3$.

\section{Discussion}

Over the last decade, there has been a hot debate about the superiority of CRT and ST as DIOPS for treatment of advanced HNSCC $(8,23,24)$. However, a recent meta-analysis clearly demonstrated that induction chemotherapy has no 
survival benefit over CRT in advanced HNSCC (25) and therefore CRT is expected to be the future mainstay of treatment. On the other hand, it is obvious that current standard CRT with high-dose CDDP, in which $100 \mathrm{mg} / \mathrm{m}^{2}$ of CDDP is administered every three weeks, needs to be optimized to reduce the severe toxicity, which is frequently observed in both the acute and the long-term phase (11-13). In this context, the quite favorable 5-year OS (73\%) and 5-year LEDFS (69\%) observed in this CRS cohort in the present study appears to offer one solution. Thus, for those who show good response to induction CRT, total 150 (split regimen) or 160 (bolus regimen) $\mathrm{mg} / \mathrm{m}^{2}$ of CDDP may be sufficient to achieve favorable survival without impairment of laryngeal function. Interestingly these CDDP doses were considerably lower than the $200 \mathrm{mg} / \mathrm{m}^{2}$ dose recommended for maintenance of the oncological results in the standard tri-week CDDP or in the recently proposed weekly $\left(40 \mathrm{mg} / \mathrm{m}^{2}\right)$ CDDP protocol (26-28) and therefore more applicable to the elderly patients and/or patients with poor general condition. This result suggests that a majority of the CRS tumors are composed of less aggressive tumors which are amenable to cure with the moderate intensity CRT alone; this inference is also supported by the significantly lower rates of tumor-related death in the CRS arm as compared to that in the N-CRS arm. Thus, chemoradioselection provides an accurate measure of the biological aggressiveness of each tumor, and may be a useful strategy for optimization of the intensity of CRT. This method is comparable with chemoselection, which entails one or a few cycles of induction chemotherapy as a tool to select patients for organ preservation $(20,21,29)$. However, given the conclusions of the above-mentioned meta-analysis (25) and the time- and cost-effectiveness, chemoradioselection is thought to be a better method than chemoselection.

In this study, the premise of VALCSG and EORTC $(1,2)$, i.e., DIOPS is a better option than radical surgery in patients with advanced laryngeal and hypopharyngeal cancer, because of better quality of life and comparable survival rates, was questioned. The N-CRS-ope showed markedly better 5-year OS $(70 \%)$ at the expense of the larynx, which otherwise remained at $47 \%$ as shown by the N-CRS-ref, which indicates that under selected conditions, radical surgery confers survival benefit over CRT. Because similar rates of distant metastases were observed in these two populations, the rates of loco-regional failure appear to be the main cause of this difference; the N-CRS-refu demonstrated as high as the $32 \%$ of loco-regional failure, which probably caused by the high local recurrence $(56 \%)$ and poor salvage $(43 \%)$, whereas in the N-CRS-ope arm the incidence of loco-regional failure was only $9 \%$. Interestingly, only refusal of surgery (and not $\mathrm{T}$, $\mathrm{N}$, and primary site) was an independent predictor of adverse prognosis in the N-CRS population, as was clearly demonstrated in our multivariate analysis. This result implies that independent of the tumor stage and site, chemoradioselection can accurately segregate patients who would be better treated with curative-intent surgery at a relatively early phase of treatment. Moreover, in a similar analysis of the overall population, the refusal of surgery alone correlated with increased risks of death, which validates our algorithm-based treatment decision (i.e., organ preservation vs. surgery). In the cohort of patients who were initially treated with CRT alone (i.e., the
CRS plus CRS-ref), hypopharyngeal cancer and refusal of surgery was associated with poor prognosis. The former result confirms a general fact that when treated with similar modalities hypopharyngeal cancer shows poorer prognosis than laryngeal cancer. Whereas the latter finding indicates that irrespective of the tumor stage and site, continuation of CRT for poor responders eventually culminates in poor survival. Given that this similar phenomenon has long been recognized in the field of ST and has been used as the logical basis for chemoselection $(20,21,29)$, it is quite puzzling that CRT strategy has stuck to the policy of definitive CRT and salvage surgery without adequate attention to the biological selection so far. Collectively, our results suggest that poor responders should be treated by radical surgery with curative-intent at a relatively early phase of CRT rather than by definitive CRT, which increases the risk of the local failure and eventually compromises survival.

In this algorithm-based strategy, improvement of the overall LEDFS (41\%), which is mainly worsened by poor results of T3 tumors (25\%), is an essential issue to be addressed. We altered the regimen from split to bolus CDDP, with the expectation that a substantial increase in the rate of chemoradioselection may lead to the improved results including an increase in LEDFS in T3 cases. Our preliminary data suggests that this alteration succeeded in increasing the rate of chemoradioselection and improved overall OS and LEDFS. However, in T3 cases the improvement in LEDFS was not observed. These results indicate the superiority of bolus CDDP as well as its limitation for laryngeal preservation, particularly in advanced T. It is obvious that further dose-intensification inevitably leads to the enhanced toxicity and tends to diminish the utility of chemoradioselection. Thus, addition of a molecular-targeted approach to our strategy seems to be a rational method to address this problem. Recently we found that induction of a putative cancer stem cell marker, CD44 variant 9 (CD44v9), was a major hurdle to chemoradioselection. Thus, addition of CD44v9 targeting (e.g., sulfasalazine) to chemoradioselection may be a promising approach to enhance the efficacy of chemoradioselection and thereby improve survival and laryngeal preservation (30).

In conclusion, our algorithm-based 'chemoradioselection' might provide a novel platform for the treatment of advanced HNSCC and help optimize the currently employed excessive treatment intensity. We have started a multi-institutional prospective study to verify the utility of this protocol on a larger scale.

\section{Acknowledgement}

The present study was supported in part by fund from Grants-in-Aid for Scientific Research (C): 24592600 to Muneyuki Masuda.

\section{References}

1. Department of Veterans Affairs Laryngeal Cancer Study Group, Wolf GT, Fisher SG, Hong WK, Hillman R, Spaulding M, Laramore GE, Endicott JW, McClatchey K and Henderson WG: Induction chemotherapy plus radiation compared with surgery plus radiation in patients with advanced laryngeal cancer. N Engl J Med 324: 1685-1690, 1991. 
2. Lefebvre JL, Chevalier D, Luboinski B, Kirkpatrick A, Collette $\mathrm{L}$ and Sahmoud T: Larynx preservation in pyriform sinus cancer: Preliminary results of a European organization for research and treatment of cancer phase III trial. EORTC Head and Neck Cancer Cooperative Group. J Natl Cancer Inst 88: 890-899, 1996.

3. Posner MR, Hershock DM, Blajman CR, Mickiewicz E, Winquist E, Gorbounova V, Tjulandin S, Shin DM, Cullen K, Ervin TJ, et al: Cisplatin and fluorouracil alone or with docetaxel in head and neck cancer. N Engl J Med 357: 1705-1715, 2007.

4. Vermorken JB, Remenar E, van Herpen C, Gorlia T, Mesia R, Degardin M, Stewart JS, Jelic S, Betka J, Preiss JH, et al: Cisplatin, fluorouracil, and docetaxel in unresectable head and neck cancer. N Engl J Med 357: 1695-1704, 2007.

5. Adelstein DJ, Saxton JP, Rybicki LA, Esclamado RM, Wood BG, Strome M, Lavertu P, Lorenz RR and Carroll MA: Multiagent concurrent chemoradiotherapy for locoregionally advanced squamous cell head and neck cancer: Mature results from a single institution. J Clin Oncol 24: 1064-1071, 2006.

6. Forastiere AA, Goepfert H, Maor M, Pajak TF, Weber R, Morrison W, Glisson B, Trotti A, Ridge JA, Chao C, et al: Concurrent chemotherapy and radiotherapy for organ preservation in advanced laryngeal cancer. N Engl J Med 349: 2091-2098, 2003.

7. Hanna GJ, Haddad RI and Lorch JH: Induction chemotherapy for locoregionally advanced head and neck cancer: Past, present, future? Oncologist 18: 288-293, 2013.

8. Haddad R, O'Neill A, Rabinowits G, Tishler R, Khuri F, Adkins D, Clark J, Sarlis N, Lorch J, Beitler JJ, et al: Induction chemotherapy followed by concurrent chemoradiotherapy (sequential chemoradiotherapy) versus concurrent chemoradiotherapy alone in locally advanced head and neck cancer (PARADIGM): A randomised phase 3 trial. Lancet Oncol 14: 257-264, 2013.

9. Hoffman HT, Porter K, Karnell LH, Cooper JS, Weber RS, Langer CJ, Ang KK, Gay G, Stewart A and Robinson RA: Laryngeal cancer in the United States: Changes in demographics, patterns of care, and survival. Laryngoscope 116 (9 Pt 2 Suppl 111): S1-S13, 2006.

10. Newman JR, Connolly TM, Illing EA, Kilgore ML, Locher JL and Carroll WR: Survival trends in hypopharyngeal cancer: A population-based review. Laryngoscope 125: 624-629, 2015.

11. Machtay M, Moughan J, Trotti A, Garden AS, Weber RS, Cooper JS, Forastiere A and Ang KK: Factors associated with severe late toxicity after concurrent chemoradiation for locally advanced head and neck cancer: An RTOG analysis. J Clin Oncol 26: 3582-3589, 2008

12. Argiris A, Brockstein BE, Haraf DJ, Stenson KM, Mittal BB, Kies MS, Rosen FR, Jovanovic B and Vokes EE: Competing causes of death and second primary tumors in patients with locoregionally advanced head and neck cancer treated with chemoradiotherapy. Clin Cancer Res 10: 1956-1962, 2004.

13. Corry J, Peters LJ and Rischin D: Optimising the therapeutic ratio in head and neck cancer. Lancet Oncol 11: 287-291, 2010.

14. Kumamoto Y, Masuda M, Kuratomi Y, Toh S, Shinokuma A, Chujo K, Yamamoto T and Komiyama S: 'FAR' chemoradiotherapy improves laryngeal preservation rates in patients with T2N0 glottic carcinoma. Head Neck 24: 637-642, 2002.

15. Masuda M, Kamizono K, Uryu H, Fujimura A and Uchi R: Roles of therapeutic selective neck dissection in multidisciplinary treatment. Journal, 2012. In: Neck Dissection - Clinical Application and Recent Advances. In Tech, Croatia, pp49-60, 2012

16. Hirata K, Horikoshi N, Aiba K, Okazaki M, Denno R, Sasaki K, Nakano Y, Ishizuka H, Yamada Y, Uno S, et al: Pharmacokinetic study of S-1, a novel oral fluorouracil antitumor drug. Clin Cancer Res 5: 2000-2005, 1999.
17. Nonoshita T, Shioyama Y, Nakamura K, Nakashima T, Ohga S, Yoshitake T, Ohnishi K, Terashima K and Asai K: Concurrent chemoradiotherapy with S-1 for T2N0 glottic squamous cell carcinoma. J Radiat Res 51: 481-484, 2010.

18. Ohnishi K, Shioyama Y, Nakamura K, Nakashima T, Ohga S, Nonoshita T, Yoshitake T, Terashima K, Komune S and Honda H: Concurrent chemoradiotherapy with S-1 as first-line treatment for patients with oropharyngeal cancer. J Radiat Res 52: 47-53, 2011.

19. Masuda M, Matsuo M, Aso T, Kiyohara H, Rikimaru F, Kunitake N and Higaki Y: Utility of algorithm-based chemoradioselection in the treatment for advanced hypopharyngeal carcinoma. Head Neck 37: 1290-1296, 2015.

20. Strojan P, Haigentz M Jr, Bradford CR, Wolf GT, Hartl DM, Langendijk JA, Rinaldo A, Eisbruch A, Mendenhall WM, Forastiere AA, et al: Chemoradiotherapy vs. total laryngectomy for primary treatment of advanced laryngeal squamous cell carcinoma. Oral Oncol 49: 283-286, 2013.

21. Forastiere AA, Weber RS and Trotti A: Organ preservation for advanced larynx cancer: Issues and outcomes. J Clin Oncol 33: 3262-3268, 2015.

22. Lefebvre JL and Ang KK; Larynx Preservation Consensus Panel: Larynx preservation clinical trial design: Key issues and recommendations-a consensus panel summary. Int J Radiat Oncol Biol Phys 73: 1293-1303, 2009.

23. Cohen EE, Karrison TG, Kocherginsky M, Huang $\mathrm{CH}$, Agulnik M, Mittal BB, Yunus F, Samant S, Brockstein B, Raez LE, et al: DeCIDE: A phase III randomized trial of docetaxel (D), cisplatin (P), 5-fluorouracil (F) (TPF) induction chemotherapy (IC) in patients with N2/N3 locally advanced squamous cell carcinoma of the head and neck (SCCHN). J Clin Oncol 30 (suppl): S5500, 2012.

24. Hitt R, Grau JJ, Lopez-Pousa A, López-Pousa A, Berrocal A, García-Girón C, Irigoyen A, Sastre J, Martínez-Trufero J, Brandariz Castelo JA, et al: A randomized phase III trial comparing induction chemotherapy followed by chemoradiotherapy versus chemoradiotherapy alone as treatment of unresectable head and neck cancer. Ann Oncol 25: 216-225, 2014.

25. Blanchard P, Landais C, Petit C, et al: Meta-analysis of chemotherapy in head and neck cencer (MACH-NC): an update on 100 randamized traials and 19,248 patients, on behalf of MANH-HN group. ESMO Meeting abstract, 9500, 2016

26. Ang KK: Concurrent radiation chemotherapy for locally advanced head and neck carcinoma: Are we addressing burning subjects? J Clin Oncol 22: 4657-4659, 2004.

27. Melotek JM, Cooper BT, Koshy M, Silverman JS and Spiotto MT: Weekly versus every-three-weeks platinum-based chemoradiation regimens for head and neck cancer. J Otolaryngol Head Neck Surg 45: 62, 2016.

28. Ho KF, Swindell R and Brammer CV: Dose intensity comparison between weekly and 3-weekly Cisplatin delivered concurrently with radical radiotherapy for head and neck cancer: A retrospective comparison from New Cross Hospital, Wolverhampton, UK. Acta Oncol 47: 1513-1518, 2008.

29. Urba S, Wolf G, Eisbruch A, Worden F, Lee J, Bradford C, Teknos T, Chepeha D, Prince M, Hogikyan N and Taylor J: Single-cycle induction chemotherapy selects patients with advanced laryngeal cancer for combined chemoradiation: A new treatment paradigm. J Clin Oncol 24: 593-598, 2006

30. Aso T, Matsuo M, Kiyohara H, Taguchi K, Rikimaru F, Shimokawa M, Segawa Y, Higaki Y, Umeno H, Nakashima T and Masuda M: Induction of CD44 variant 9-expressing cancer stem cells might attenuate the efficacy of chemoradioselection and Worsens the prognosis of patients with advanced head and neck cancer. PLoS One 10: e0116596, 2015. 